\title{
Highlights from Compass in hadron spectroscopy
}

\author{
Fabian Krinner for the Compass collaboration ${ }^{1, a}$ \\ ${ }^{1}$ Technische Universität München, Physik-Department, E18
}

\begin{abstract}
Since Quantum Choromdynamics allows for gluon self-coupling, quarks and gluons cannot be observed as free particles, but only their bound states, the hadrons. This so-called confinement phenomenon is responsible for $98 \%$ of the mass in the visible universe. Measurement of the hadron excitation spectra therefore gives valuable input for theory and phenomenology to quantitatively understand this phenomenon.

One simple model to describe hadrons is the Constituent Quark Model (CQM), which knows two types of hadrons: mesons consisting of a quark and an antiquark and baryons, which are made of three quarks. More advanced models, which are inspired by QCD as well as calculations within Lattice QCD, predict the existence of other types of hadrons, which may be, e.g., described solely by gluonic excitations (glueballs) or mixed quark and gluon excitations (hybrids).

In order to search for such states, the Compass experiment at the Super Proton Synchrotron at CERN has collected large data sets, which allow to study the light-quark meson and baryon spectra with unmatched precision. The overview shown here focuses on the light meson sector, presenting a detailed Partial-Wave Analysis of the processes: $\pi^{-} p \rightarrow \pi^{-} \pi^{+} \pi^{-} p$ and $\pi^{-} p \rightarrow \pi^{-} \pi^{0} \pi^{0} p$. A new state, the $a_{1}(1420)$ with $J^{P C}=1^{++}$, is observed. Its Breit-Wigner parameters are found to be in the ranges: $m=1412-1422 \mathrm{MeV} / c^{2}$ and $\Gamma=130-150 \mathrm{MeV} / c^{2}$. In the same analysis, a signal in a wave with $J^{P C}=1^{-+}$is observed. A resonant origin of this signal would not be explicable within the CQM. In addition to this possibility of an exotic state, possible non-resonant origin of this signal is discussed.
\end{abstract}

\section{The Compass spectrometer}

The multi-purpose fixed-target spectrometer CoMPAss is located at CERN's northern area and is supplied with secondary hadron or tertiary muon beams by the Super Proton Synchrotron. The two-stage spectrometer setup allows for a wide physics program, which includes, e.g., studies of the spin structure of the nucleon as well as hadron spectroscopy, which will be presented here.

For the analysis shown here, data taken in the year 2008 is used. There a $190 \mathrm{GeV} / c$ negative hadron beam, composed of $\pi^{-}(97 \%)$ with some minor contributions from $K^{-}(2 \%)$ and antiprotons (1\%), impinged on a $40 \mathrm{~cm}$ long liquid hydrogen target.

The analysis of this data, which is the subject of this article, was performed on two three-pion final states, $\pi^{-} p \rightarrow$ $\pi^{-} \pi^{0} \pi^{0} p$ and $\pi^{-} p \rightarrow \pi^{-} \pi^{+} \pi^{-} p$. For these channels, $3.5 \cdot 10^{6}$ and $50 \cdot 10^{6}$ events were recorded in the neutral and charged channel, respectively. The charged data set is the largest for this particular process.

The analyses of both channels were performed independently of each other using different software packages. The systematic uncertainties of both channels differ, since the reconstruction relies on different parts of the spectrometer.

Nevertheless, physics is the same in both channels, which allows for an effective cross-check of the results.

\section{Analysis method}

For the studies presented here, a detailed Partial-Wave Analysis (PWA) was performed on both diffractively produced three-pion final states. In this class of processes, an incoming $\pi^{-}$from the beam gets excited via an interaction with the target and forms an intermediate state $X^{-}$. In our analysis, this interaction is assumed to be dominated by Pomeron exchange. This intermediate state then decays into the observed final-state particles.

The excited pion state $X^{-}$is characterized by the quantum numbers $J^{P C} M^{\epsilon}$, where $J$ gives the spin, $P$ and $C$ the eigenvalues of the parity and generalized charge conjugation operators, $M$ the magnetic quantum number and $\epsilon$ the reflectivity.

Since the Pomeron exchange does not uniquely determine the quantum numbers, there are many possible values they can take and which can all interfere with each other. Therefore, the main goal of the PWA is to disentangle all appearing contributions of intermediate states with different $J^{P C} M^{\epsilon}$.

\subsection{The isobar-model}

Since the considered processes end up in three-particle final-states, the isobar model is employed in the PWA. Here the assumption is made that the excited states do

\footnotetext{
ae-mail: fabian-krinner@mytum.de
} 


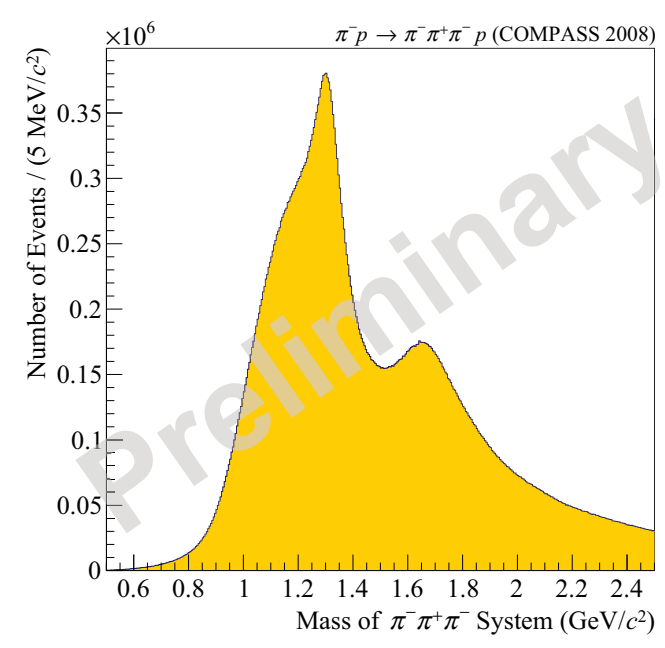

Figure 1. Invariant mass distribution of the $\pi^{-} \pi^{+} \pi^{-}$ final state.

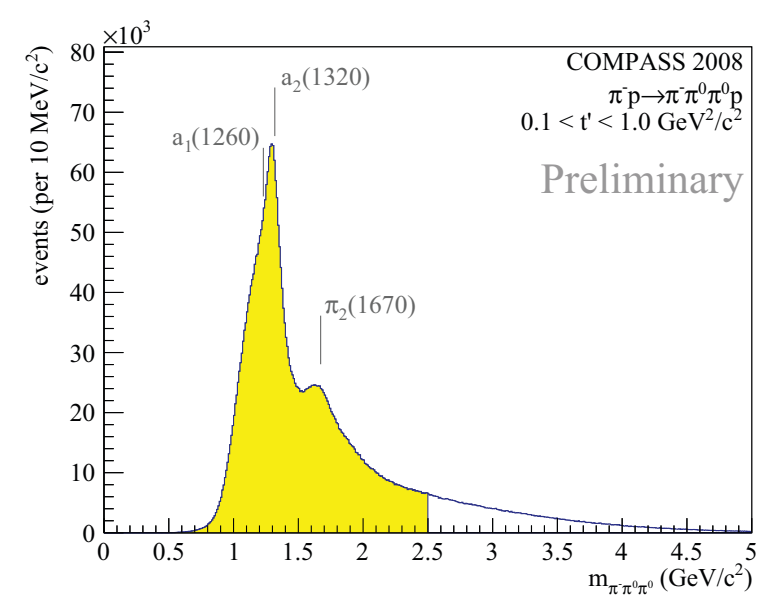

Figure 2. Invariant mass distribution of the $\pi^{-} \pi^{0} \pi^{0}$ final state. Labels mark the main resonances described in sec. 3.1, which give the gross features of the spectrum.

not decay directly into the final-state particles, but perform consecutive two-particle decays into final-state particles and/or other intermediate states, the isobars. In the case at hand, $X^{-}$decays into a bachelor $\pi$ and an isobar $\xi$, which then decays into the other two pions.

An advantage of the isobar model is the factorization of production and decay of $X^{-}$. Therefore the intensity $\mathcal{I}$, the square of the complex amplitude $\mathcal{A}$, which can be expanded into a series of partial waves, can be written as:

$$
\mathcal{I}\left(m_{X}, \tau\right)=\left|\mathcal{A}\left(m_{X}, \tau\right)\right|^{2}=\left|\sum_{\text {waves }} T_{\text {wave }}\left(m_{X}\right) \psi_{\text {wave }}(\tau)\right|^{2}
$$

Here, the decay amplitudes $\psi_{\text {wave }}(\tau)$, depending on the phase-space variables $\tau$, describe the kinematic distribution of the final-state particles, where the production amplitudes $T_{\text {wave }}$ describe the production of different intermediate states $X^{-}$with given $J^{P C} M^{\epsilon}$. Within the isobar model,

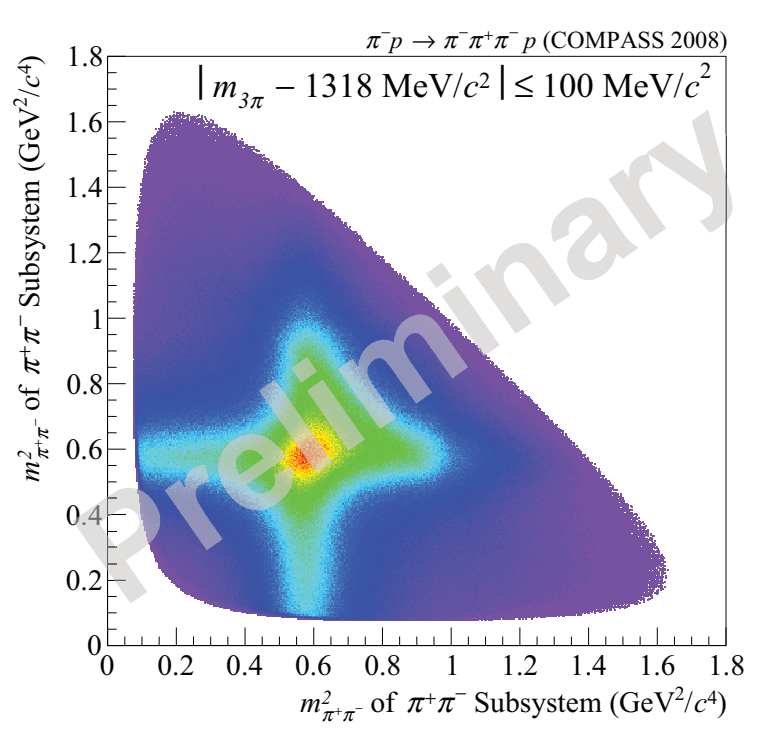

Figure 3. Dalitz plot for the $\pi^{-} \pi^{+} \pi^{-}$channel, with $m_{3 \pi}$ chosen around the mass of the $a_{2}(1320)$

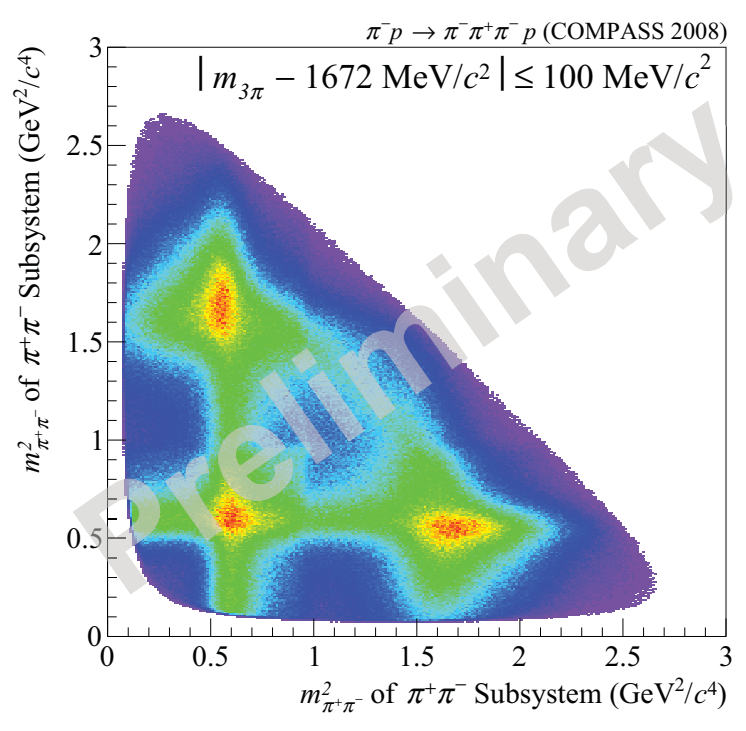

Figure 4. Dalitz plot for the $\pi^{-} \pi^{+} \pi^{-}$channel, with $m_{3 \pi}$ chosen around the mass of the $\pi_{2}(1670)$

the decay amplitudes $\psi(\tau)$ can be calculated, putting known complex parametrizations for the line shapes for the isobars into the fit. In our analysis, the following isobars were used [1-3]:

\begin{tabular}{l|l}
$I^{G} J^{P C}$ & \\
\hline $0^{+} 0^{++}$ & {$[\pi \pi]_{S}, f_{0}(980), f_{0}(1500)$} \\
$1^{+} 1^{--}$ & $\rho(770)$ \\
$0^{+} 2^{++}$ & $f_{2}(1270)$ \\
$1^{+} 3^{--}$ & $\rho_{3}(1690)$
\end{tabular}

With the $\psi(\tau)$ known, the production amplitudes $T\left(m_{X}\right)$ can be extracted from the data by fitting the intensity of eq. (1) to the data in bins of the invariant three-pion mass $m_{3 \pi}=m_{X}$. With this method, no assumptions regarding three-pion resonances have to be made. 


\subsection{The wave set}

In the amplitude parametrization of equation (1) a sum over a given set of waves appears, which are defined by:

$$
J^{P C} M^{\epsilon}[\text { isobar }] \pi L,
$$

where $J^{P C} M^{\epsilon}$ give the quantum numbers of the state $X^{-}$, while the rest describes its decay mode. Since the isobars are all well-known states, their quantum numbers are not explicitly stated in the formula above. $L$ is the relative orbital angular momentum between the isobar and the bachelor pion.

In the analyses presented, a set of 87 waves with a spin $J$ and angular momentum $L$ up to six was used. In addition, one incoherent isotropic wave was added in order to be able to describe uncorrelated events [3].

\section{Selected results}

\subsection{The biggest waves}

The main features of the invariant mass spectra of both channels shown in Figs. 3 and 4 can be explained by the three biggest waves in the analysis, namely:

- $1^{++} 0^{+} \rho(770) \pi S$, Fig. 5: This wave describes an axialvector intermediate state decaying into $\rho(770)$ and a pion. With $33 \%$ of the intensity in the charged channel, this wave is the biggest in the analysis. The dominant structure visible is the $a_{1}(1260)$ resonance. Good agreement between the neutral and charged channels can be seen.

- $2^{++} 1^{+} \rho(770) \pi D$, Fig. 6: This wave is the second biggest wave in the analysis, taking $8 \%$ of the total intensity. It describes a spin-2 meson, which also decays into $\rho(770) \pi$. In this wave, the clearest three-pion resonance, the $a_{2}(1320)$ can be seen, again with good agreement between both channels.

- $2^{-+} 0^{+} f_{2}(1270) \pi S$, Fig. 7: The third biggest wave gives rise to the second peak in the invariant mass spectra. It describes an intermediate state behaving like a pion with spin 2 decaying into $f_{2}(1270) \pi$. The main resonance visible is the $\pi_{2}(1670)$. This wave takes $7 \%$ of the charged channel intensity.

The Partial-Wave Analysis performed is not just able to extract the gross features of the spectra, but can resolve contributions down to the sub-percent level. One of these waves is $4^{++} 1^{+} \rho(770) \pi G$, depicted in Fig. 8, which describes a spin- 4 meson decaying into $\rho(770) \pi$. This wave takes only about $0.76 \%$ intensity, but the $a_{4}(2040)$ resonance can be clearly seen in both channels.

\subsection{The $\mathrm{a}_{1}(1420)$}

Besides the well known resonances shown up to now, a new resonance, the $a_{1}(1420)$, was seen in the $1^{++} 0^{+} f_{0}(980) \pi P$ wave. The corresponding intermediate state has the same quantum numbers as the biggest wave, but it decays via a peculiar decay mode, $f_{0}(980) \pi$.

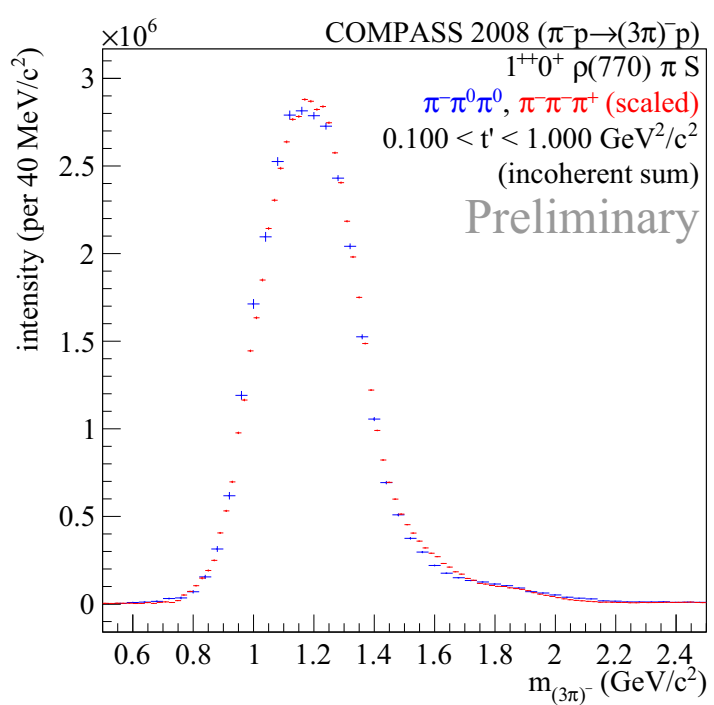

Figure 5. Intensity of the $1^{++} 0^{+} \rho(770) \pi S$ wave for both three-pion channels. The intensity of the charged channel is scaled to match the integral of the neutral channel.

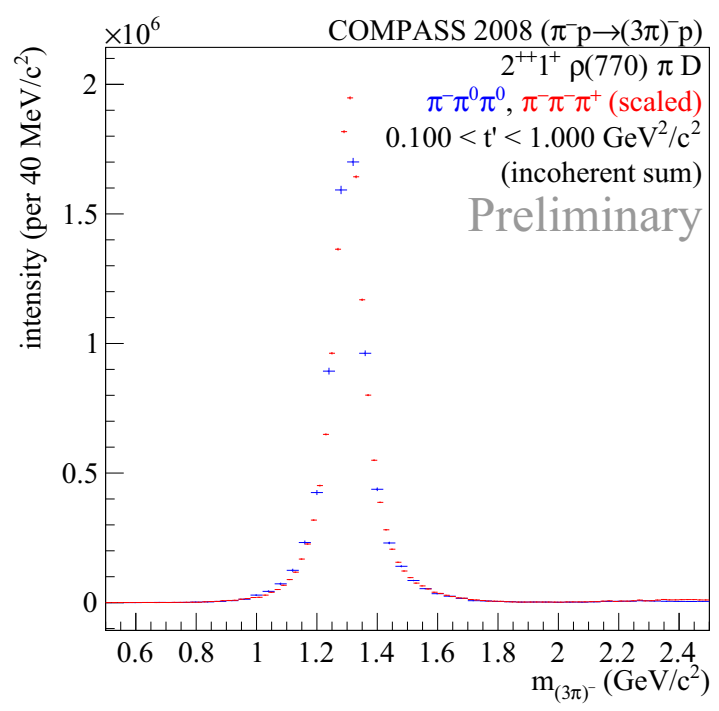

Figure 6. Intensity of the $2^{++} 1^{+} \rho(770) \pi D$ wave for both three-pion channels. The intensity of the charged channel is scaled to match the integral of the neutral channel.

This new resonance appears in both channels shown in Fig. 9. A clear and rapid phase motion can be seen with respect to the biggest wave in the model, depicted in Fig. 10. This indicates that the observed intensity peak constitutes an actual resonance. The extracted Breit-Wigner parameters, mass and width, lie in the following ranges:

$$
\begin{aligned}
m & =1412-1422 \mathrm{MeV} / c^{2} \\
\Gamma & =130-150 \mathrm{MeV} / c^{2}
\end{aligned}
$$

Observation of a new resonance in this mass region is interesting for a number of reasons. First, no model or lattice QCD predicted a resonance in the discussed mass region. Second, the $a_{1}(1420)$ decays into $f_{0}(980) \pi$ with an unusually small relative intensity compared to other resonances. 


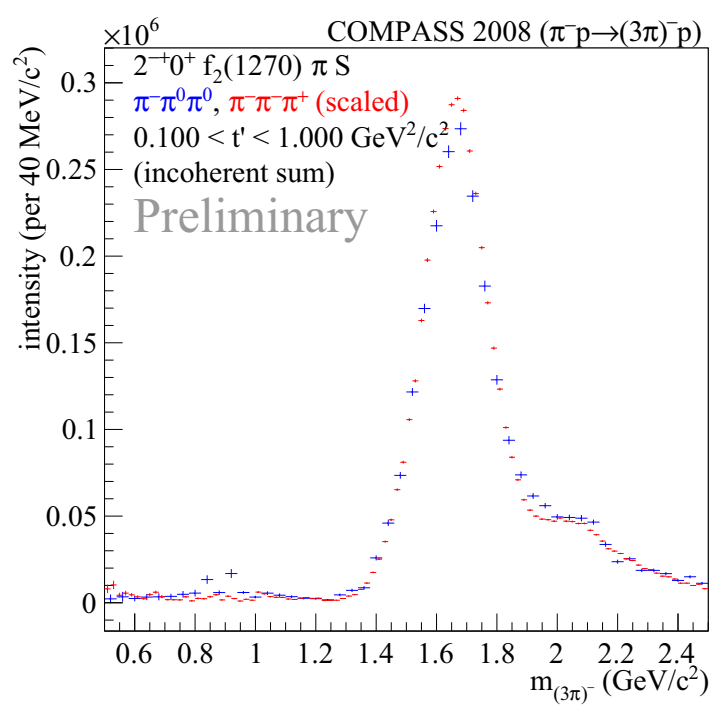

Figure 7. Intensity of the $2^{-+} 0^{+} f_{2}(1270) \pi S$ wave for both three-pion channels. The intensity of the charged channel is scaled to match the integral of the neutral channel.

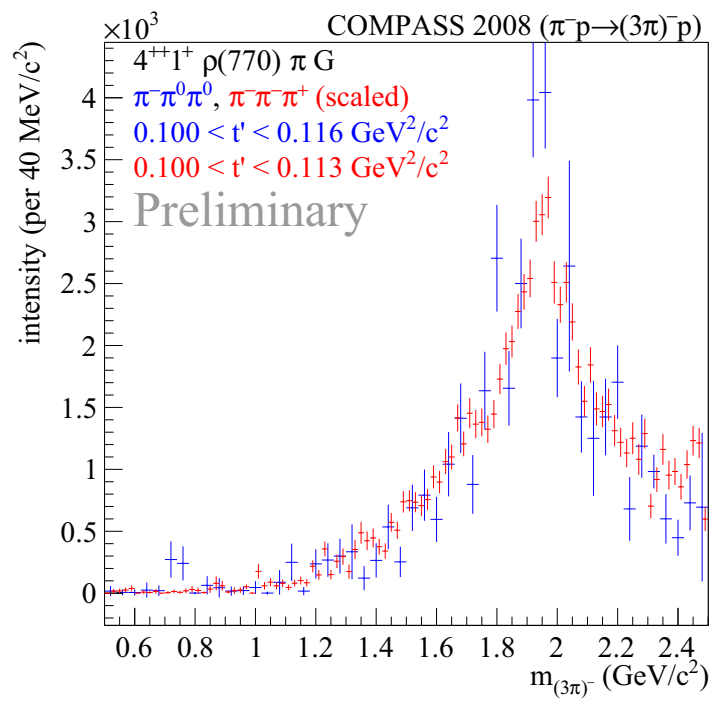

Figure 8. Intensity of the $4^{++} 1^{+} \rho(770) \pi G$ wave for both three-pion channels. The intensity of the charged channel is scaled to match the integral of the neutral channel.

Additionally, the $f_{0}(980)$ is also known to strongly couple to $K \bar{K}$ states. Third, the mass of the $a_{1}(1420)$ is only slightly bigger than the $K \bar{K}^{*}$ threshold. This might be a hint for a dynamic nature of this resonance involving $K \bar{K}^{*}$ loop diagrams, but the true nature of the $a_{1}(1420)$ remains unclear at the moment [4].

\subsection{Spin exotic signal}

In addition to the waves shown previously, which all had non-exotic quantum numbers, i.e., they can be explained within the Constituent-Quark Model (CQM), a signal has been observed in the $1^{-+} 1^{+} \rho(770) \pi P$ wave. Due to its exotic quantum numbers $J^{P C}=1^{-+}$, thus behaving like a

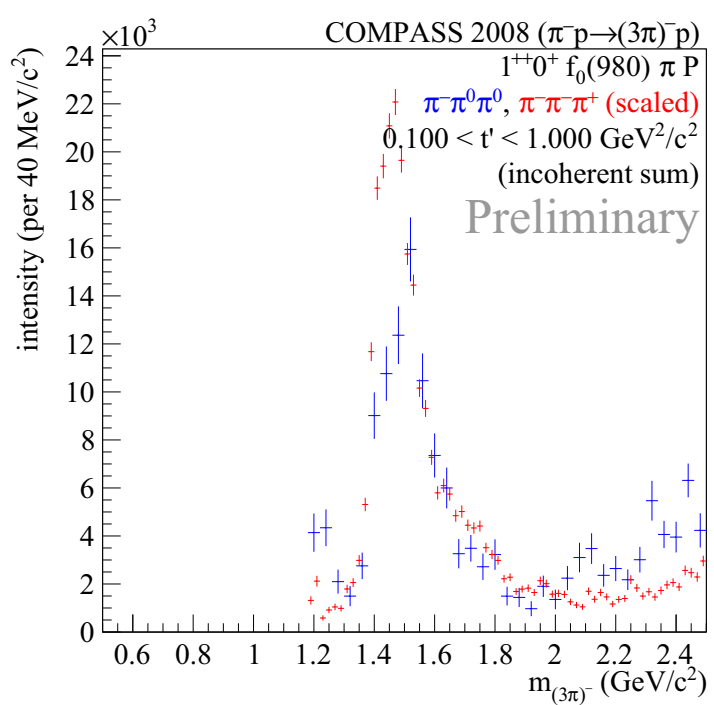

Figure 9. Intensity of the $1^{++} 0^{+} f_{0}(980) \pi P$ wave. The peak of the $a_{1}(1420)$ is clearly visible in both channels.

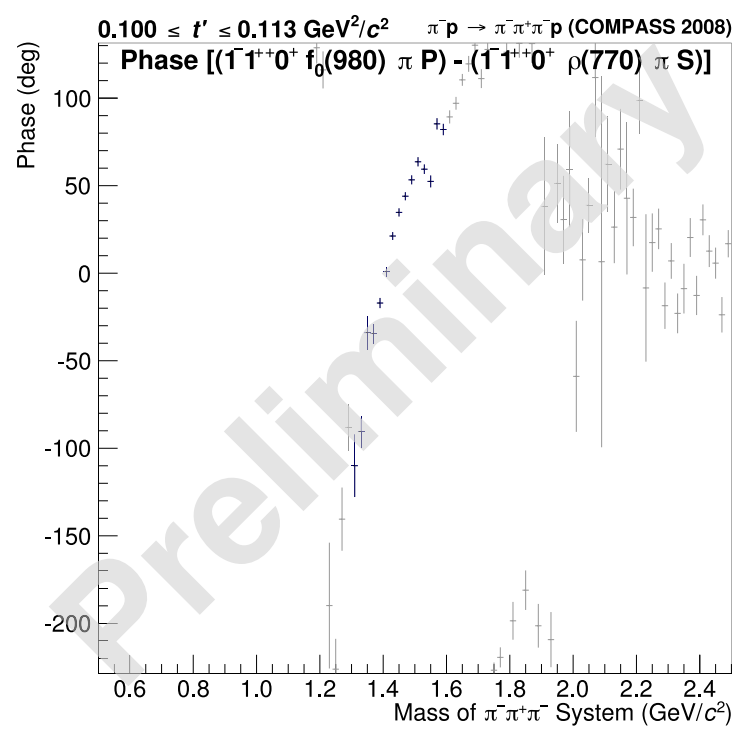

Figure 10. Relative phase between the $1^{++} 0^{+} \rho(770) \pi S$ and $1^{++} 0^{+} f_{0}(980) \pi P$ waves. In the mass region of the $a_{1}(1420)$, a clear phase motion is visible.

pion with spin 1, a resonance in this wave would exceed the frame of the CQM. As in the previous waves, the signal is seen in the charged as well as the neutral channel.

Besides a possible resonance, there exist other, nonresonant mechanisms that can lead to a signal in the discussed wave. One of these mechanisms is the so-called Deck effect, which is diagrammatically shown in Fig. 12. To study its effect, PWA was performed on pseudo-data generated with the Deck model for the charged channel. The results of the Deck's Partial-Wave decomposition are then compared with the results for real data.

To further disentangle such effects, this study is also per- 


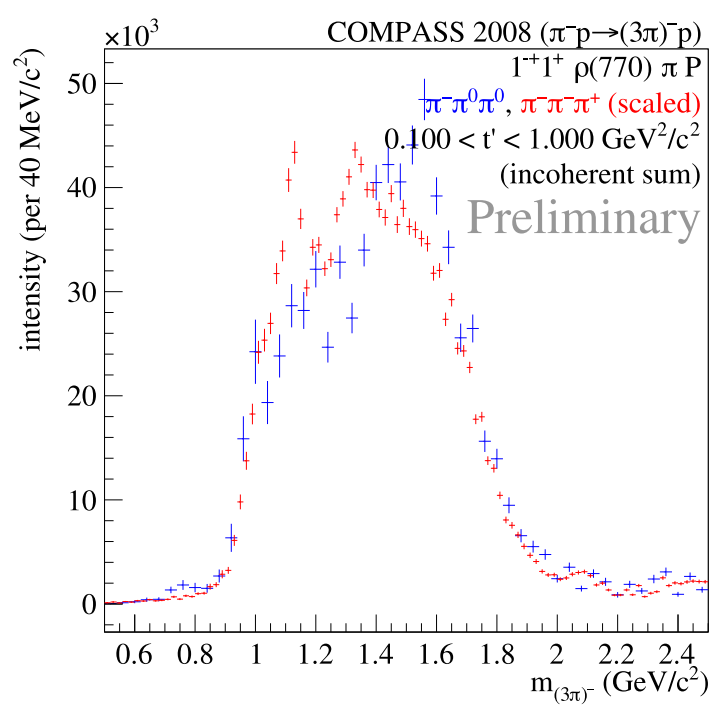

Figure 11. Intensity of the spin exotic $1^{-+} 1^{+} \rho(770) \pi P$ wave. A similar signal is visible in both cahnnels.

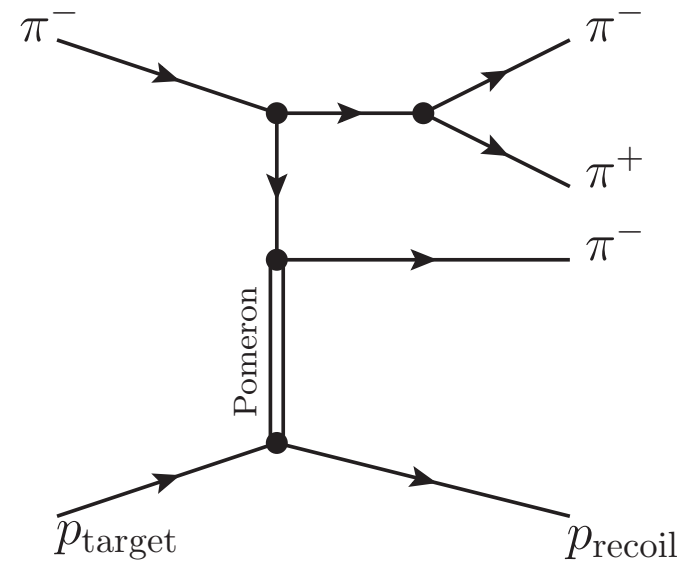

Figure 12. Diagrammatic view of the non-resonant Deck effect. In this process, no three-pion intemediate state appears.

formed in bins of $t^{\prime}$, with:

$$
t^{\prime}=|t|-\left|t_{\min }\right| \simeq-t,
$$

where $t$ is the four-momentum transfer between a beam pion and target proton. This allows to better disentangle resonant and non-resonant contributions since the resonance parameters may not depend on $t^{\prime}$.

\section{Conclusions}

Due to large three-pion data sets collected by Compass, very detailed Partial-Wave Analysis could be performed, allowing for a systematic cross check between the two
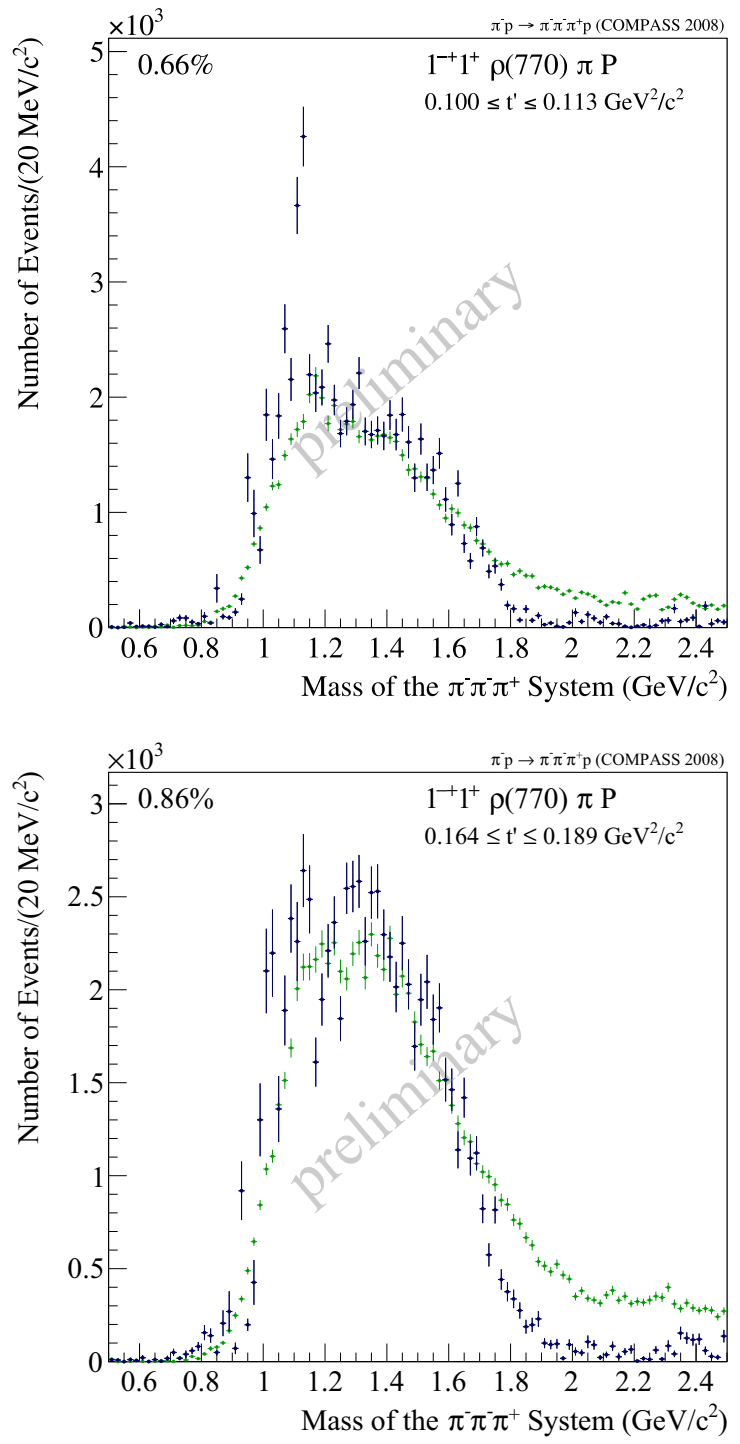

Figure 13. Intensity distribution for the spin exotic wave for the data (blue) and the Deck Monte-Carlo (green), normalized to the integrals over mass and $t^{\prime}$, at low values of the four-momentum transfer. The intensity in the data can be explained well by the Deck effect.

channels and thus giving deep insight into the spectrum of light mesons.

For the present analysis a large wave set, comprising of 87 waves up to spin 6 was employed, and waves contributing only at the sub-percent level to the total intensity could be extracted. Besides reproducing all well-known resonances with isospin $I^{G}=1^{-}$, a previously unknown state, the $a_{1}(1420)$, was extracted in the $1^{++} 0^{+} f_{0}(980) \pi P$ wave. In addition to these resonances, which all have quantum numbers that can be explained by the CQM, a signal in the exotic-spin $1^{-+} 1^{+} \rho(770) \pi P$ wave was seen. Possible, non-resonant origin of this signal, the Deck effect, was studied and compared to the data. With the Deck effect, the signal corresponding to low four-momentum transfer can be explained well, while for high $t^{\prime}$ it shows an excess over the Deck decomposition. 

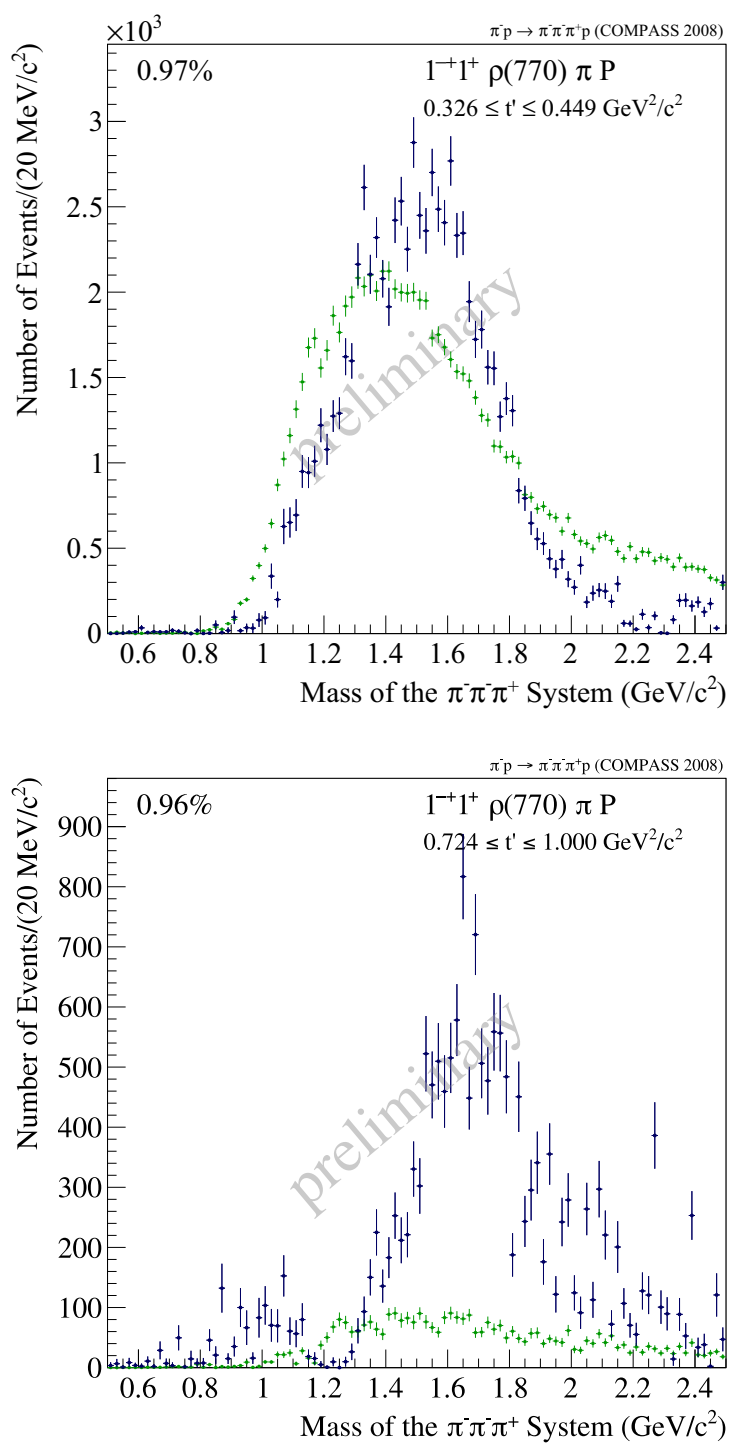

Figure 14. Intensity distribution for the spin exotic wave for the data (blue) and the Deck Monte-Carlo (green), normalized to the integrals over mass and $t^{\prime}$, at high values of the four-momentum transfer. The Deck contribution vanishes, while the signal stays also at high $t^{\prime}$.

\section{Outlook}

Since the analyses presented here did not involve any line shape for the resonances, mass-dependent fits have to be performed, to determine these and thus extracting masses and widths of the appearing resonances [5].

The presented analysis still relies on the isobar model, which assumes fixed line shapes for the appearing isobars. To see, if this assumption is justified, a new method is being studied to directly extract the isobar shapes from the data. This allows to check the validity of the isobar model and to determine resonance parameters for the isobars as well.

Due to the large number of events, non-resonant contributions also become important. One of them, the Deck contribution introduced above, is expected to have big impact on the data. To study its particular influence on the PWA will be the aim of further studies.[6]

\section{References}

[1] J. Beringer et al. [Particle Data Group Collaboration], Phys. Rev. D 86 (2012) 010001.

[2] K. L. Au, D. Morgan and M. R. Pennington, Phys. Rev. D 35 (1987) 1633.

[3] F. Haas, Two-Dimensional Partial-Wave Analysis of Exclusive $190 \mathrm{GeV} \pi^{-} p$ Scattering into the $\pi^{-} \pi^{-} \pi^{+}$ Final State at Compass (CERN), PhD Thesis, Technische Universität München, 2014.

[4] J. L. Basdevant and E. L. Berger, Phys. Rev. D 16 (1977) 657.

[5] F. Haas, Proceedings of the 13th International Workshop on Meson Production, Properties and Interaction (2014), Kraków, Poland, EPJ Web of Conferences 81, 01004 (2014).

[6] F. Krinner, Proceedings of the 52nd International Winter Meeting on Nuclear Physics (2014), Bormio, Italy, PoS(Bormio 2014)031. 